\title{
Design of a Site-Built Integrated Collector Storage Solar Water Heater Under Uncertainty
}

\author{
Eve Commerford ${ }^{1}$, Patrick L. Gurian ${ }^{1}$, Jin Wen ${ }^{1}$ and Steve R. Cook $^{2}$ \\ ${ }^{1}$ Department of Civil, Architectural, and Environmental Engineering, Drexel University, Philadelphia, PA 19104, USA \\ ${ }^{2}$ Energy Center, The University of Texas El Paso, El Paso, TX 79968, USA
}

\begin{abstract}
A straightforward framework for identifying key design parameters and determining their values for a site-built integrated collector storage solar water heater (ICSSWH) under uncertainty is developed. The framework is then used to evaluate the design for a ICSSWH, which uses locally available materials and can be assembled by builders without specialized training in solar system construction. In the framework, first the most important design parameters are identified by a sensitivity analysis using TRNSED software to predict system performance. Then a decision tree is constructed that identifies discrete outcomes of various combinations of design parameters, uncertainty quantities, and resulting system performance as predicted by the TRNSED model. For the ICSSWH that is evaluated in this project, three key design parameters are identified: storage tank size, top surface area, and household daily hot water cosumption. Three different system sizes are evaluated in this manner, a 114-liter, 227-liter, and 341-liter system. Only the 341-liter system has a favorable economic return compared to a conventional, gas heater. All systems present some risk of performing worse than a conventional system. Further research is warranted into higher surface area designs to improve the performance of the system. The expected return of the system selected by this analysis is modestly higher than the return of the option which would be selected without any consideratoin of uncertainty. This indicates that straightforward frameworks, such as this, for considering uncertainty in the design of residential solar water heaters, may offer benefits to builders and home buyers.
\end{abstract}

\section{INTRODUCTION}

In the United States, $21 \%$ of primary energy, including $23 \%$ of natural gas and $36 \%$ of electricity, is consumed by residential houses, which is more than the energy consumed by commercial buildings (17\% of primary energy) [1]. Among all residential appliances, space heating consumes $31.5 \%$ and water heating consumes $12.6 \%$ of primary energy. These costs are particularly burdensome for lowincome households who spend a disproportionate amount of their income on basic utilities. While on average American families spend 3.5\% of their budget on energy consumption, low income families spend $14 \%$ of their budget [2]. Utilizing alternative energy, especially solar energy, is a sustainable option for low-income families to reduce their utility costs. However, market-available systems that utilize alternative energy often have high initial and installation costs, which make them less applicable for low-income families. Another approach is to install very low-cost site-built systems which utilize alternative energy, such as site-built integrated collector storage passive solar water heaters (ICSSWH). In this design, the same container is used for heating and storing the water. This is clearly not ideal from an energy transfer point of view as a collector should have high surface area and a storage tank low surface area. However, it avoids the need for two separate containers and simplifies the design and construction. Packaged ICSSWH systems are commercially available, but a site-built approach, in which the materials,

*Address correspondence to this author at the Department of Civil, Architectural, and Environmental Engineering, Drexel University, Philadelphia, PA 19104, USA; Tel: 215-895-2889; Fax: 215-895-1363; E-mail: plg28@drexel.edu such as storage tank and glazing material, are obtained offthe-shelf and locally, can reduce the overall cost even further. Another way to reduce the cost is to design the system so that it can be assembled by builders without specialized training in solar technologies using locally available materials. Because the builders are not specialized in solar technologies, the parameters for the installed systems may be subject to uncertainties. For example, the slope of the collector may not be installed exactly as designed. Similarly, the hot water demand is uncertain because hot water consumption varies from household to household. Because these values are not known precisely in advance, the design parameters should be chosen so that the system performs well regardless of how these uncertainties are resolved.

Smyth et al. [3] reviewed the literature which discusses ICSSWH design. From the review, it is observed that existing studies mostly focus on optimizing packaged ICSSWHs. Few studies provide guidance about how to design a sitebuilt ICSSWH, especially an ICSSWH system built from locally available materials. ICSSWHs are subject to uncertainty in demand, gas prices, performance, etc. This is a complex decision for designers, builders, and homeowners. Although systematic decision analysis processes have been evaluated on some HVAC system designs by a few researchers $[4,5]$, there is a lack of systematic methodology for ICSSWH design under uncertainty.

Such a design methodology should be based on lifecycle costs, which are the present value of the fossil fuel savings achieved by the system over its entire life, less the initial capital outlay for the construction of the system. While a wide range of sophisticated analytical tools are available for decision making under uncertainty, the design process for a 


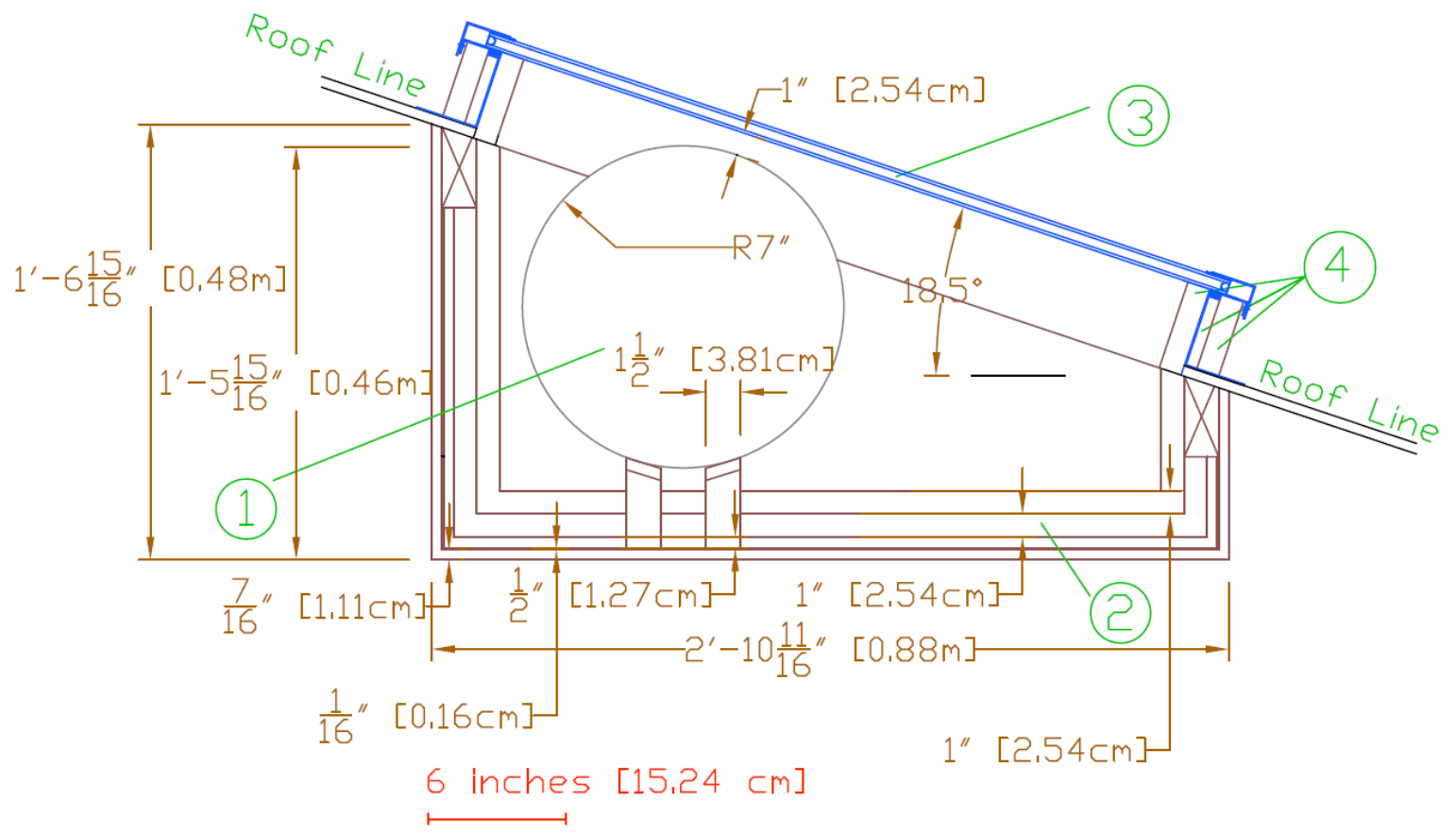

Fig. (1). Side view of the ICSSWH under evaluation (the collector/storage tank (1), collector box (2) and patio door glazing (3)).

residential application should be simple and transparent to the homeowner and builder.

A related question is how risk may influence solar hot water heater design. For example, the appropriate sizing of the system is dependent on fossil fuel costs. Systems designed to optimize performance under current fossil fuel prices might be oversized if fossil fuel prices decline or undersized if fossil fuel prices increase. Different decision makers may have different risk tolerances. In particular, risk averse decision makers may prefer to invest in a solar water heater as a potential hedge against higher fossil fuel costs in the future, even if expected returns are not highly favorable. However, there is a need for a straightforward framework that will let homeowners evaluate the risks and returns of investments in renewable energy technologies.

Therefore, the objectives for this project are: 1) Develop a straightforward framework for the design of site-built ICSSWH under uncertainty; 2) Evaluate the performance of the site-built ICSSWH design using this framework; 3) Assess the importance of including uncertainty in the design of this hot water heater; and 4) Identify how different risk tolerances may influence the preferred design alternative.

In this paper, the site-built ICSSWH system, to which this decision framework is to be applied, is introduced in Sec. 2. Section 3 presents the decision analysis methodology that is developed for ICSSWH design with uncertainties. Section 4 discusses the results generated from the analysis methodology presented in Section 3. Conclusions from this project are provided in Section 5.

\section{SYSTEM PROFILE}

The ICSSWH that is under evaluation for this project is a roof-integrated passive system. Design parameters and costs for the base case system analyzed here are based on the design used in a technology transfer program in El Paso, Texas.
Fig. (1) provides the side view, as well as dimensions for the system. Each household has two side-by-side collector boxes each of which contains a 114-liter storage tank and a glazing surface. The storage tank has a non-selective black surface and can be easily obtained from local building suppliers. The glazing surface for a collector box is made from the glass of a dissembled patio door with a total surface area of $2.7 \mathrm{~m}^{2}$. However, as shown in Fig. (1), only part of the total surface area is directly above the storage tank. The effective area of the glazing surface is considered as $1.2 \mathrm{~m}^{2}$, which is the glazing area directly above the storage tank. This is a conservative estimation of the effective glazing surface area because some solar energy entering into the box from the glazing that is not directly above the tank may be absorbed by the storage tank after reflection by the box interior surface. The effect of glazing surface area on total system performance is evaluated in the following section. The height of the collector enclosure above the roof line is 8.8 centimeters. The slope and azimuth of the collector are 18.5 degrees and zero degrees respectively. The slope of the collector matches the slope of the roof line to make the system more aesthetically pleasing. An auxiliary 151 liter liquid propane water heater is used. The energy factor and recovery efficiency for the auxiliary heater are 0.56 and 0.78 respectively and the rated input is 4.2 Megajoule/hr. The set point hot water delivery temperature is $51.7^{\circ} \mathrm{C}$. The system described above is considered as the base system for this project.

\section{METHODS}

There are many design parameters that can affect the overall system performance. Developing a framework to identify the most important parameters and to assess the impact of parameter uncertainty on the overall system costeffectiveness is the objective here. The framework proposed in this study integrates a computer model of the physical system with an assessment of expected economic returns of 
Table 1. Parameters for TRNSED Analysis Test 1-1.1 Input Parameters

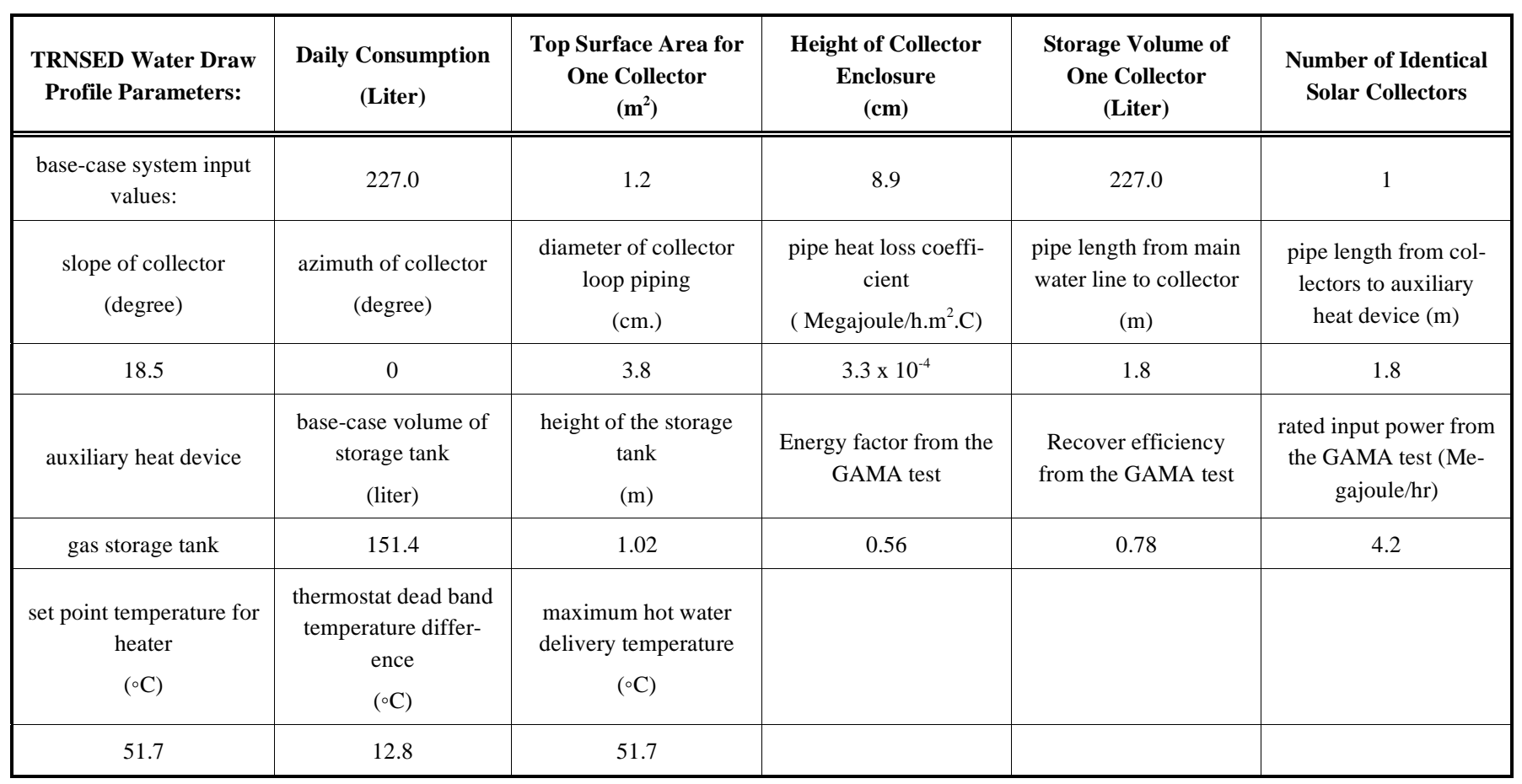

different designs under uncertainty. The TRNSED [6] computer model can be used to simulate the monthly expected hot water production and gas savings for particular designs and climate conditions. These performance measures output from the TRNSED model are input into an economic analysis model that estimates the net present value of the investment in the solar water heater. A sensitivity analysis is then conducted to identify key design parameters for which an uncertainty analysis is merited. The uncertainty analysis is conducted with a decision tree approach implemented in PrecisionTree software [7]. Each of these components of the analysis is described in more detail in the following sections.

In this study the framework is developed and demonstrated for a single location, El Paso, TX. However, the TRNSED program contains a library of climatic data which allows the performance of different designs to be simulated in any part of the U.S. To implement this approach in a different location, it would first be necessary to run the TRNSED program using the climatic data appropriate for the location under consideration. The remainder of the analysis could then be repeated using the TRNSED output for the particular location.

\subsection{TRNSED Analysis}

TRNSED is able to model two types of solar water heaters, a glycol system and an integral-collector-storage system. In this project, a non-selective surface integral-collectorstorage system is simulated. A base system is defined first, as described in Sec. 2. Input parameters for the base system are summarized in Table 1-1.1. Each simulation is conducted for one full year using average weather conditions for El Paso, TX. Output values from a simulation of this base-case system are summarized in Table 1-1.2.

\subsection{Economic Analysis and Sensitivity Assessment}

The savings per year in the TRNSED Analysis software is given in terms of energy. The energy savings are converted to dollars at a rate of US\$0.012/Megajoule based on a quote from the El Paso plumbing association of US\$0.32/ liter for liquid propane [8]. Annual gas costs are assumed to increase by a given percentage each year, which constitutes a geometric annuity. The present value of a geometric annuity is given by Eq. 1:

$$
P=A *\left[\frac{1-(1+g)^{n}(1+i)^{-n}}{(i-g)}\right]
$$

where $P$ is the projected savings on gas consumption; $A$ is the current annual savings on gas consumption; $g$ is the growth rate; $i$ is the interest rate (set to 7\%) and $n$ is the number of years the system will be used (assumed to be 20). Lifecycle costs are the savings less the capital costs of the system, US\$700 for a 114-liter system, US\$1,200 for a 227liter system, and US\$1400 for a 341-liter system based on current costs from an ongoing pilot program.

To identify the most important parameters for the uncertainty analysis, a one-way sensitivity analysis was conducted. In this analysis, plausible ranges of different model parameters were first identified [9]. Separate model runs were conducted with each variable in turn set first at its upper bound and then at its lower bound (and the remaining variables set at their base-case values). Table 2 shows the base-case values and range for each parameter in the oneway sensitivity analysis. Upper and lower bounds are chosen to be roughly representative of the $95^{\text {th }}$ and $5^{\text {th }}$ percentile values of these quantities. 


\section{1-1.2 Output Parameters}

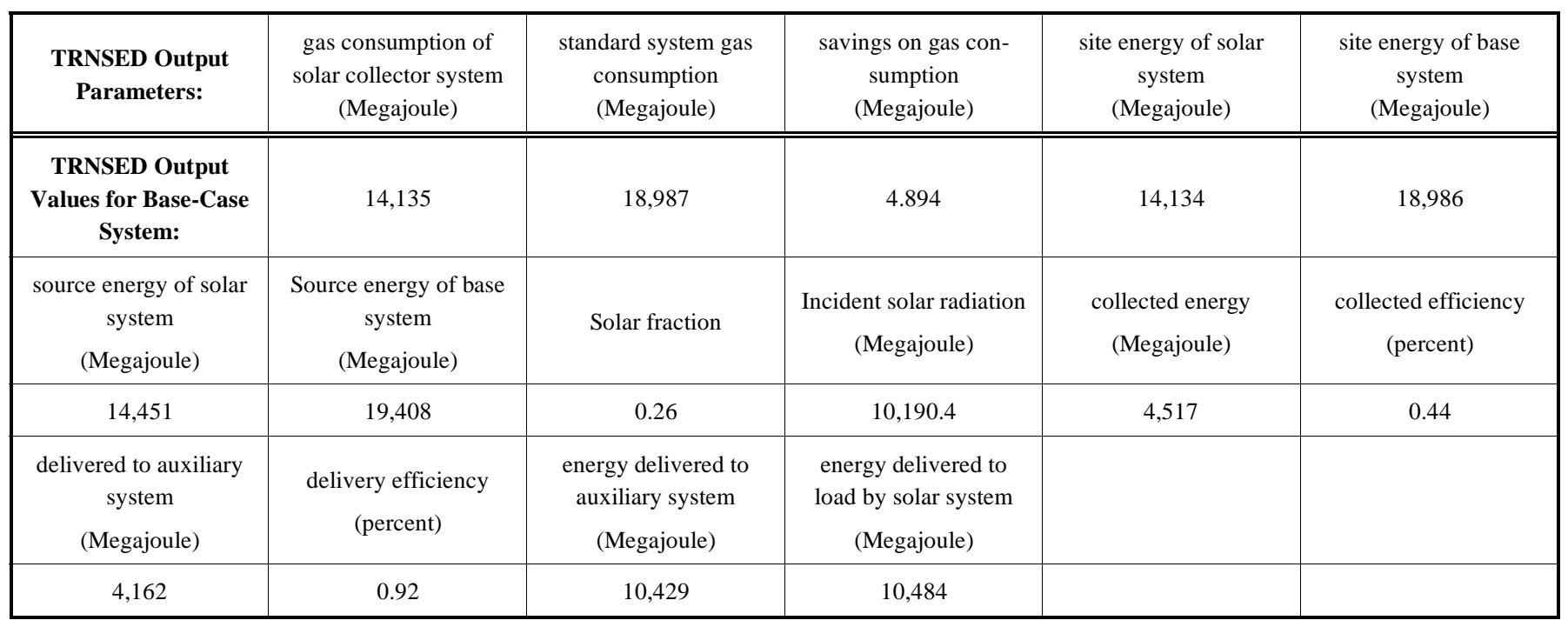

Table 2. Parameters Analyzed

\begin{tabular}{|c|c|c|c|c|}
\hline Parameter Input & Base-Case Value & Units & Lower Bound & Upper Bound \\
\hline Daily consumption & 227 & Liters & 151 & 303 \\
\hline Volume of storage section & 227 & Liters & 114 & 341 \\
\hline Slope of collector & 18.5 & Degrees & 9 & 31.45 \\
\hline Pipe heat loss coefficient & $3.3 \times 10^{-4}$ & Megajoule/h.m².C & $1.3 \times 10^{-3}$ & $6.6 \times 10^{-4}$ \\
\hline Set point temperature for heater & 51.7 & deg. C & 48.9 & 54.4 \\
\hline Maximum hot water delivery temperature & 51.7 & deg. C & 48.9 & 54.4 \\
\hline
\end{tabular}

NA-lower bound not analyzed.

\subsection{Decision Tree Analysis}

A decision tree, constructed with the PrecisionTree software, was used to show the potential design choices and major uncertainties. The process of developing the decision tree is shown in Fig. (2). Rectangular nodes represent decisions (i.e., factors under the control of the designer), and circular nodes, known as chance nodes, represent uncertain events. Discrete probabilities are assigned to continuously varying uncertain quantities using the Pearson-Tukey method [7] in which the $5^{\text {th }}$ and $95^{\text {th }}$ percentiles are each assigned probabilities of 0.185 and the median is assigned a probability of 0.63. (For a normal random variable, this discrete approximation preserves the mean and variance.) The decision tree is then analyzed to identify the option with the highest expected value. This analysis is conducted by successively reducing nodes (i.e., replacing the branches coming from a node with an equivalent single value) from the right to the left of the tree. Chance nodes are reduced by calculating the expected value of the outcomes. Decision nodes are reduced by taking the maximum value of the different branches. At each decision node, the branch with the highest expected value is noted as the preferred option. This process is continued until the last node on the left is reached, at which point the decision or sequence of decisions that maximize the expected value of the possible outcomes has been identified.

The decision tree solution process identifies the option with the highest expected value, given the uncertainties modeled in the tree. One can also identify the option which would have been picked if, instead of calculating expected values, one had ignored the uncertainty in the outcomes and based the decision only on the most likely outcomes. In this case, chance nodes are reduced by taking the value of the branch with the highest probability. The decision made in this manner may or may not differ from the decision made when uncertainty is considered (i.e., expected values are taken at chance nodes). If the decision does not change, then one may plausibly conclude that there is no benefit to considering uncertainty in this case, and that a simple analysis based on most likely values is sufficient to reach the appropriate design decisions. If the decision does change, then one can compare the expected value of the decision made by considering uncertainty with the expected value of the deci- 


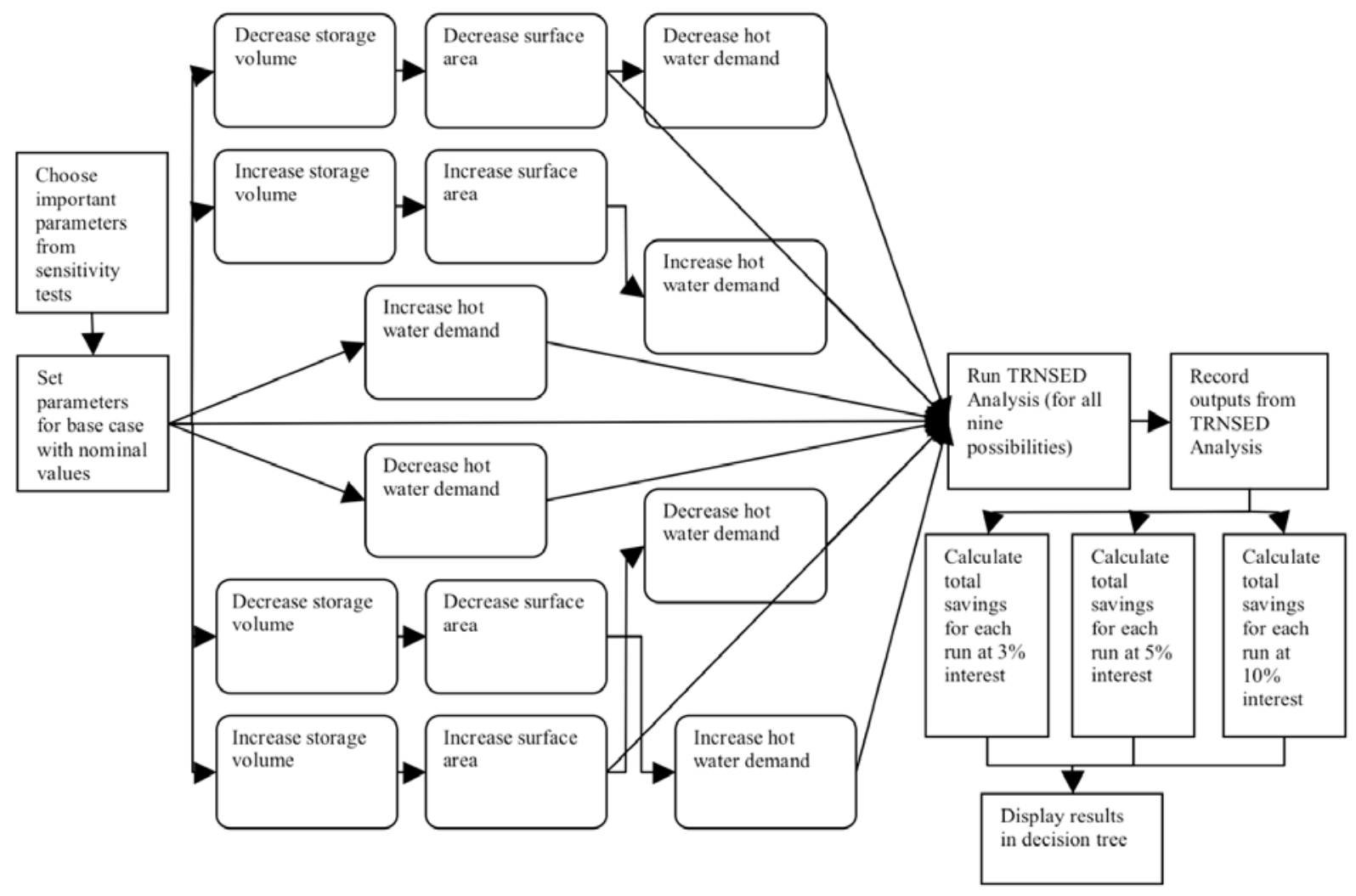

Fig. (2). Flow chart for creating decision tree.

sion which is made when uncertainty is neglected. The improvement in the expected results is termed the expected value of including uncertainty, provides insight into how important it is to conduct an uncertainty analysis for the particular decision problem [10]. When outcomes are distributed symmetrically about the most likely outcome, the expected value of a chance node does not differ from its most likely value. As a result uncertainty can be neglected, and chance nodes replaced with their most likely outcome. However, in cases where one outcome is very favorable or unfavorable, the expected value will differ from the most likely value, and there is potential value to considering uncertainty.

\subsection{Process Chart}

Fig. (3) summarizes the process for the proposed framework as described in the above sections.

\section{RESULTS \& DISCUSSION}

Fig. (4) shows the results of the one-way sensitivity analysis. Each row shows an input parameter and the percentage change in Megajoules saved from the base case when the indicated parameter is changed from the base case value to its lower bound value (indicated by the light gray bar) and its upper value (indicated by the dark gray bar). The surface area of the collector and the annual cost increase for gas are the two parameters with the most influence on the system performance, followed by the daily hot water demand. These three variables were selected for more detailed analysis. In addition, the collector surface area is linked to the water storage volume because the locally available tanks come in a fixed size. Collector volume and surface area are varied in parallel by using different integer numbers of 114- liter tanks. The pipe heat loss coefficient, slope of collector, and set point temperature all had less than a $10 \%$ impact on the model output and were treated as fixed quantities for the remainder of this analysis.

Fig. (5) shows the portion of the total demand (blue bar) provided by the solar system for different size systems (114, 227 , and 341 liter systems are considered corresponding to 1 , 2 , and 3 of the locally available water tanks placed in series) and for different water demands $(151,227$, and 303 liters/day). For the base case (227 liter system, 227 liters/day demand) the solar heater provides $26 \%$ of the energy required for the household's hot water (4,852 Megajoule per year compared to a total demand of 18,990 Megajoule). Using the larger system (341 liter capacity) increases the amount of energy supplied by the solar system but only modestly to $34 \%$ (6,540 Megajoule of 18,990 Megajoule). When demand is lower, the solar system provides less absolute energy but is able to meet a somewhat greater percentage of the demand. Overall these values are fairly low given that most water heaters are reported to supply 30 to 80 percent of daily demand [11]. As noted in the one-way sensitivity analysis presented above and in previous literature [3], available surface area is a key performance variable. Because all material for this ICSSWH system has to be easily obtained locally, the tank cannot be custom designed to achieve as high a ratio between the water storage tank and the net glazing area as the literature recommends. However, the box does include additional glazing area not directly above the tank. As discussed in Sec. 2, some of the solar energy entering the glazing in areas not over the tank will eventually be absorbed by the collector. As a conservative measure, in this 


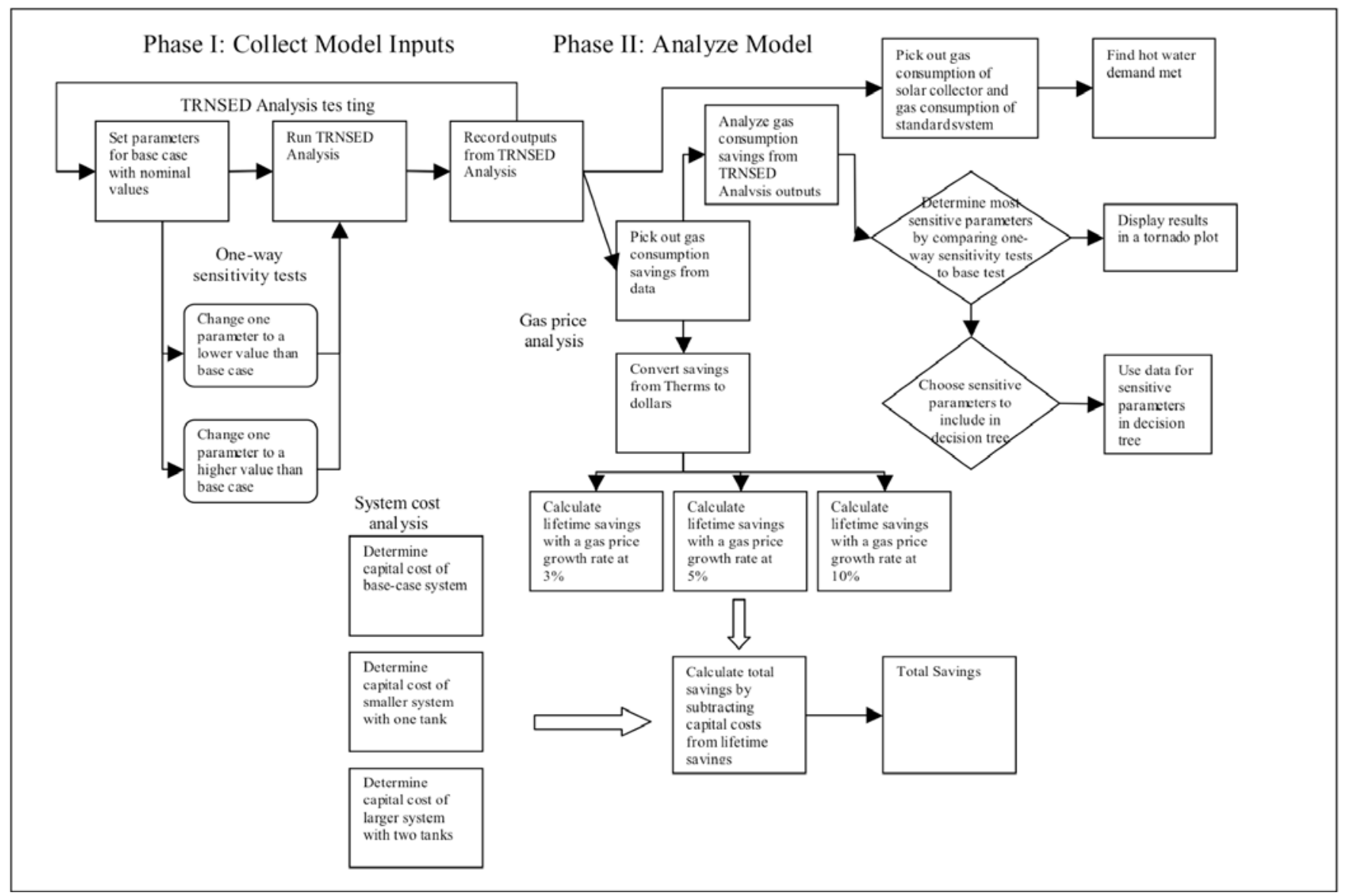

Fig. (3). Flow chart of analysis.

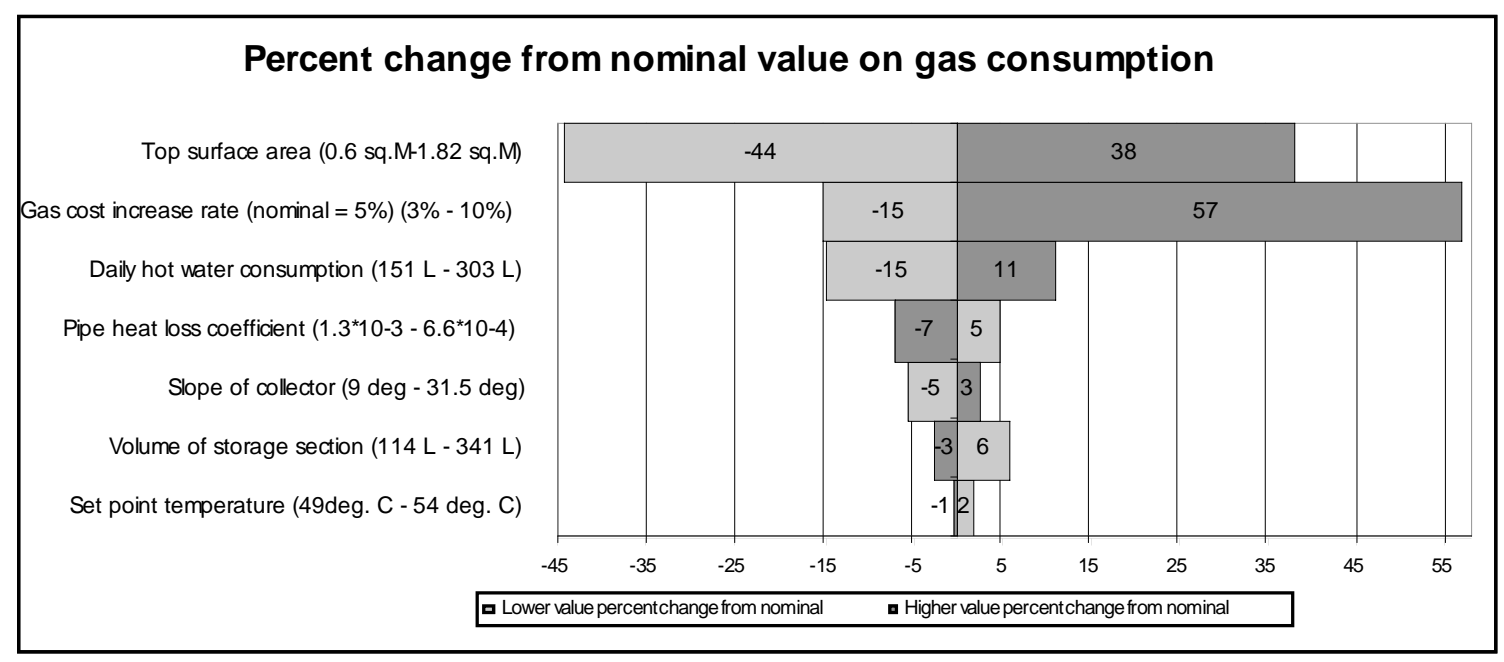

Fig. (4). One-way sensitivity analysis, bars show the expected increase or decrease in therms saved compared to base case (parameter ranges and base case values given in Table 1).

analysis the portion of the glazing area that is not directly above the storage tank is not counted as net glazing area.

Fig. (6) shows the decision tree representation of the design problem. The variables found to be important in the one-way sensitivity analysis are incorporated as variable quantities (i.e., decision or chance nodes), while the remaining model inputs are fixed at their base-case values (Table
1.1). The three possible system sizes $(114,227$, and 341 liters), along with the three hot water consumption rates (151, 227, and 303 liters/day), and three rates of increase in gas costs $(3 \%, 6 \%$, and 10\%) produce twenty seven different possible outcomes for present value of lifecycle savings relative to a conventional water heater over a 20 -year project period. 


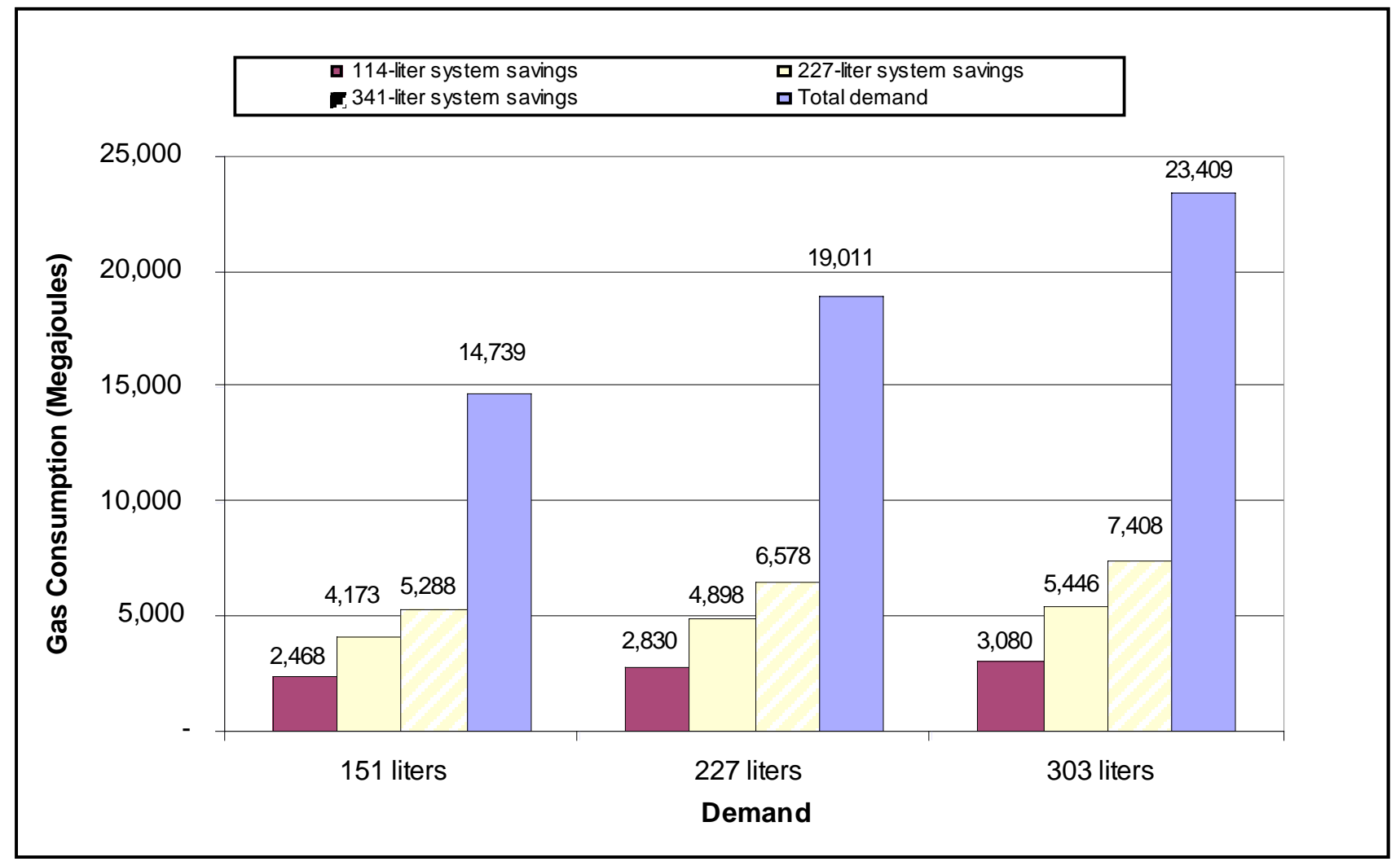

Fig. (5). Gas savings with varying hot water demand and tank size. The blue bars show the total demand while the remaining bars show the amount provided by the solar system. The difference between total demand and amount supplied by the solar system is provided by a back up, liquid propane heater.

Of the three storage volume/surface area combinations (indicated by the rectangles on the branches from the square decision node at the left of the tree), the expected value of savings is positive only for the 341 liter system (expected savings of US\$28). The expected costs for the 114 liter and 227 liter systems exceed the conventional system cost by US\$39 and US\$174, respectively. In general, the solar system is preferred to the conventional system only in the cases where gas costs increase at a $10 \%$ annual rate (although some savings are realized for the large system under high demand, when gas costs increase at 5\% annually).

A consumer considering purchasing such a system is likely to be concerned with not just expected return but also the risk of losing money relative to a conventional system. It is notable that the option preferred based on expected value, the 341-liter system, also presents the risk of the greatest loss (this system has a loss of US $\$ 530$ when both demand and gas cost increases are low). As further designs are explored, this approach can be applied and a priority placed on identifying a system with positive returns under all likely scenarios.

To investigate the importance of uncertainty on the decision, one first identifies option which would be selected if the decision were based on the most likely scenario, rather than the expected value analysis described above. The 114 liter system would then be assigned a return of -US\$58, corresponding to the 227 liter demand and $5 \%$ gas cost increase branches for this system. Similarly the 227 liter system would be assigned a return of -US\$209 and the 341-liter system a return of -US\$9. Under such circumstances, the decision maker would select a conventional system (savings of US\$0). However, when uncertainty is considered, the preferred decision is the 340-liter system (expected savings of US\$28) rather than the conventional system (expected savings of US\$0). Therefore, the expected value of including uncertainty in this problem is the difference between these two values (i.e., the expected value of the option selected when ncertainty is considered, less the expected value when uncertainty is neglected) or US\$28. This is a modest value, but it does suggest that including uncertainty can improve the decision. In particular reflecting on less optimistic scenarios as to gas price increases may motivate the purchase of a solar water heater that would not be justified based on a base-case analysis alone.

To revise this analysis for a different location, TRNSED is used to estimate the gas savings for each of the 3 designs (three tank sizes) under each of the 3 demand scenarios. Thus a total of 9 TRNSED runs are required. The gas savings values are then converted to present value using Equation 1 under each of the three gas cost increase scenarios, and the capital cost of the heater is subtracted from the savings. This produces 27 different scenarios, one for each branch of the tree in Fig. (6). The expected value of each of the 9 gas cost increase nodes is computed as: 

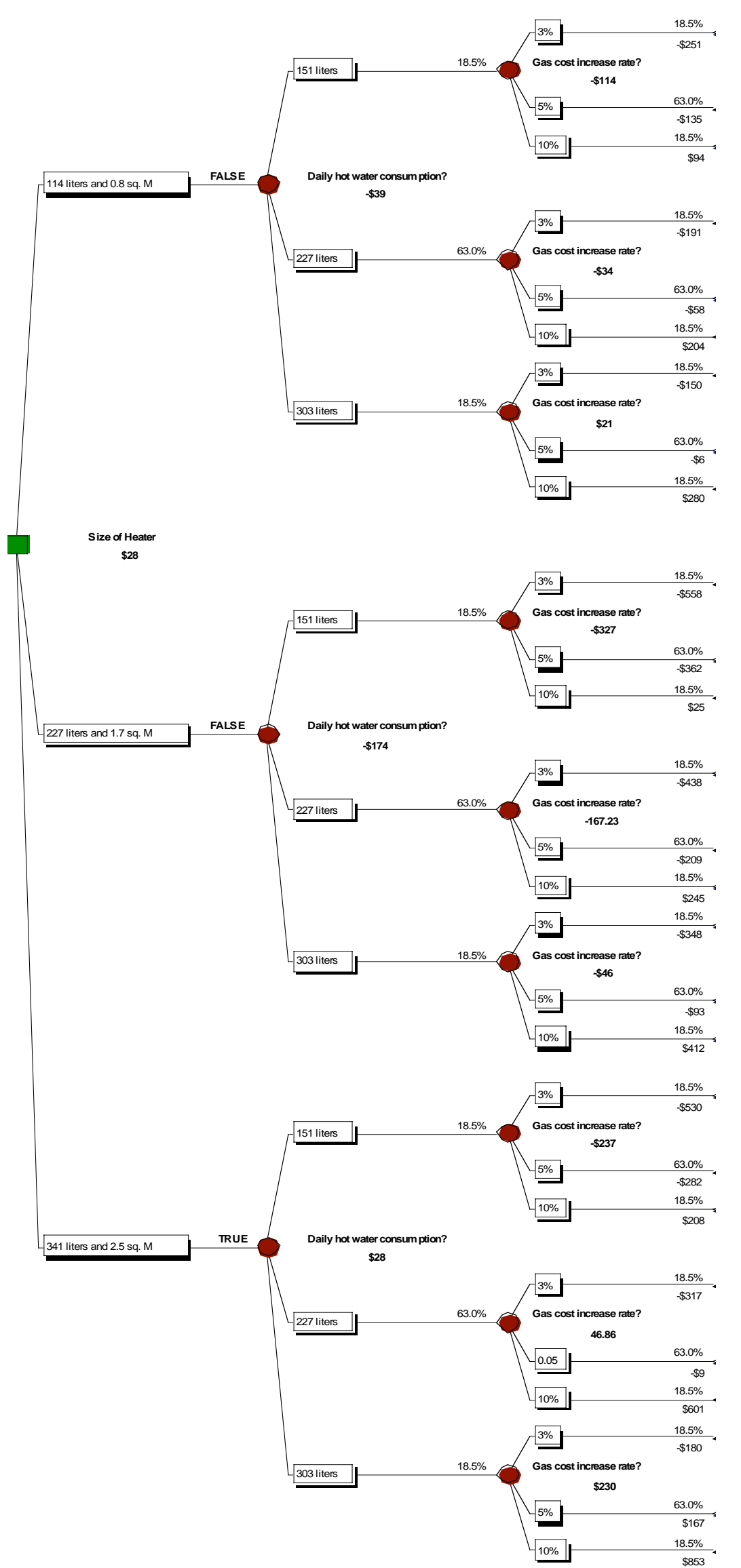

Fig. (6). Decision tree of solar water heater sizing. Expected values given to the right of each chance node. The branch with highest expected value from decision node indicated by "TRUE" with its expected value given to the right of the decision node. 


$$
\begin{aligned}
& \text { Gas node }=0.185 * \text { savings }(g=10 \%)+0.63 * \text { savings }(g=5 \%) \\
& +0.185 * \text { savings }(g=3 \%)
\end{aligned}
$$

The expected values of each of the 3 daily hot water consumption nodes are then computed by:

Water node $=0.185^{*}$ gas node $(\mathrm{D}=303)+0.63^{*}$ gas node

$(\mathrm{D}=227)+0.185^{*}$ gas node $(\mathrm{D}=114)$

where $\mathrm{D}$ is the daily hot water demand in liters. The water consumption nodes are directly after the decision node, which means that the expected values for the three decision options are equal to the expected values of their respective water consumption nodes. The designer would typically choose the design option with the highest expected value. Further research could be conducted to examine the sensitivity of the design to climatic variables and identify appropriate summary guidelines for design under uncertainty for different climatic regions.

\section{CONCLUSIONS}

A framework is developed in this study to identify key design parameters and to determine their values for ICSSWH design. The framework includes the use of ICSSWH design software, a two-way sensitivity analysis, and a decision tree analysis. The framework can also be used to evaluate how uncertainty of various design parameters may affect the overall system cost-effectiveness. The framework is then used to evaluate the design of a site-built ICSSWH design.

Using the framework, it is concluded that the 341-liter ICSSWH design considered here is slightly preferred over a conventional, gas hot water heater, while smaller designs are unfavorable relative to the conventional system. This solar system was found to be economically justified even though its performance was estimated conservatively, using a collector surface area that was based only on the glazing directly above the tank. However, designs considered here supply a relative small fraction of overall hot water demand, and present some risk of performing worse than a conventional system. Alternative designs with higher collector surface areas should be explored as these may improve the economics of the system. The inclusion of uncertainty in the analysis modestly improves the design decision, indicating that this straightforward framework for including uncertainty in residential solar hot water design may offer benefits to builders and homeowners.

\section{ACKNOWLEDGEMENTS}

This research was partially supported by National Science Foundation Awards EEC-0552792 and SES-0332001 and by the Department of Housing and Urban Development. The comment of four reviewers and Hussain Burhanpurwala's assistance with the editing of the manuscript are gratefully acknowledged.

\section{REFERENCES}

[1] DOE. Buildings Energy Data Book, US Department of Energy; United States Department of Energy: Washington, D.C. 2006.

[2] HUD. Ideas That Work - Building Communities Through Homeownership, U.S. Department of Housing and Urban Development. 2006. http://www.oup.org/files/pubs/ideasthatwork.pdf (accessed 2008).

[3] Smyth, M.; Eames, P.C.; Norton, B. Integrated collector storage solar water heaters. Renewable Sustainable Energy Rev., 2006, 10, 503-538.

[4] Wei, J.; Reddy, T. A.; Gurian, P. General methodology combining engineering optimization of primary HVAC and R plants with decision analysis methods - Part II: Uncertainty and decision analysis. HVAC\&R Res., 2007, 13 (1), 119-140.

[5] DeWit, S.; Augenbroe, G. Analysis of uncertainty in building design evaluations and its implications. Energy Build., 2002; 34 (9), 951-958. http://buildingsdatabook.eere.energy.gov/?id=view_book \#bookmark7-1 (accessed 2008).

[6] DOE. Tool Predicts Solar Domestic Water Heater Performance, United States Department of Energy. http://www.eere.energy.gov/buildings/building_america/rh_0505_t oolpredicts.html (accessed 2006).

[7] Clemen, R.T.; Reilly, T. Making hard decisions with decision tools suite; Duxbury; Pacific Grove, CA, 2001.

[8] El Paso, P. Bangor Gas Offers Burner Conversion Workshop for Heating Professionals, Plumbers El Paso.com. http://www.plumberselpaso.com/news.cfm/Article/38806/Licensed -Heating-And-Plumbing-Contractor.html (accessed 2007).

[9] Tiller, D.K.; Henze, G.P.; Guo, X. Online domestic hot water enduse database. ASHRAE Trans., 2004, 110 (2), 682-689.

[10] Morgan, M.G.; Henrion, M. Uncertainty: A Guide to Dealing with Uncertainty in Quantitative Risk and Policy Analysis; Cambridge University Press, 1990.

[11] Cook, G.; Burnett, M.S.; Delaney, C.J.; Milon, J.W.; Plowman, F.T.; Walker, K.; Wood, K.E. Energy Information Handbook Chapter II Residential Energy Use, Energy Information Document 1028. University of Florida. Gainesville, FL, 1991. http://www.p2pays.org/ref/08/07336.pdf (accessed 2008). 